\title{
Was macht eigentlich das Schweizer Gesundheitswesen so krank?
}

Daniel Schlossberg

Korrespondenz:

Dr. med. Daniel Schlossberg Bachmattstrasse 53

CH-8048 Zürich

\begin{abstract}
Einleitung
Vor etlichen Jahren erkrankten zwei renommierte Gesundheitsstrategen unerwartet. Beiden gemeinsam war, dass Herzkrankheiten auftraten, die sie zu Patienten machten, die nun plötzlich selber Leistungen des Gesundheitswesens beanspruchen mussten. Die erforderlichen Interventionen gelangen, beiden konnte nachhaltig geholfen werden. Beide änderten ihren vormals forschen Ton gegenüber der Ärzteschaft ebenso nachhaltig und hoben den hohen Standard der medizinischen Leistungen hervor - erfahren am eigenen Leib. Beide zogen sich bald aus ihren Positionen zurück und spielen heute bei der Gestaltung der Gesundheitspolitik keine Rolle mehr.
\end{abstract}

\section{Ist-Analyse:}

\section{Wer macht die Gesundheitspolitik?}

Grundsätzlich sind es zwei Kategorien von Politikern, Ökonomen und anderen Experten, die gestaltend wirken. Einerseits handelt es sich um relativ junge, (noch) gesunde Personen, die aus einer abstrakten Position heraus agieren. Sie rufen am lautesten nach Kosteneinsparungen. Andererseits sind gestandene Persönlichkeiten involviert, deren gemeinsames Merkmal darin besteht, dass sie selber zur privilegierten Schicht der Bevölkerung zählen, die medizinische Leistungen ungeachtet von Entscheidungen in der Grundversicherung nach Massgabe ihrer persönlichen Bedürfnisse uneingeschränkt beziehen.

\section{Das KVG 1996 und heute}

Vor 1996 bestand ein unbefriedigender Zustand nach Meinung der Gesundheitspolitiker, indem jeder Kanton andere Gesetze und ganz unterschiedliche Ausführungsbestimmungen kannte. Die Krankenversicherung war im wesentlichen freiwillig und nur bei Subsistenz kam ein Obligatorium zur Anwendung. Mit der Einführung des KVG wurde eine Harmonisierung gesamtschweizerisch angestrebt und ein Krankenversicherungsobligatorium eingeführt. Nach 10 Jahren KVG muss das Gesetz als gescheitert betrachtet werden. Es hat auf allen Ebenen die angestrebten Verbesserungen verfehlt. Der umfassende Leistungskatalog auf Niveau Grundversicherung mit dem Ziel, eine Zwei- oder Mehrklassenmedi- zin zu vermeiden, hat genau diese bereits eingeführt. Die Prämien sind nach Einführung des KVG überproportional angestiegen und für einen Teil der Bevölkerung nicht mehr ohne Unterstützungsleistungen durch die Kantone bezahlbar. Wie wird dieser Pyrrhussieg kommuniziert? Die Politiker suchen Sündenböcke für die Teuerung und finden diese in den Gruppierungen mit fehlender Lobby. Niemand steht hin und sucht die Fehler bei sich selber oder wenigstens in seiner Nähe.

\section{Worin besteht das Problem der fehlenden Kostenkontrolle?}

Argumentiert wird mit Mengenausweitung seitens der Leistungserbringer, ein gewisser Gesundheitsökonom spricht von den Ärzten, die «am Staatstropf» hingen. Das Bild der bösen, sich bereichernden Ärzte wird suggeriert, die zu verantworten hätten, dass die Prämien steigen. Sehen wir uns das genauer an, so erkennen wir unschwer, dass hier die «Sündenbocktheorie» herhalten muss. Sie muss das systematische Defizit in der Argumentationsfigur der Verfechter des KVG kaschieren helfen.

Wir können hier nur summarisch die Punkte aufführen, die wirklich kostenrelevant sind. Sattsam bekannt ist, dass die Lebenserwartung der Bevölkerung in den letzten Jahrzehnten markant gestiegen ist. Dies führt dazu, dass Rentenumwandlungssätze für die Ausrichtung der Pensionen angepasst werden müssen. Durch die statistische Lebensverlängerung werden Lebensjahre in Gesundheit und Krankheit hinzugewonnen. Der Zuwachs an kranken Lebensjahren steigt mit dem medizinischen Fortschritt überproportional an. Medizinisch $\mathrm{zu}$ erbringende Leistungen schnellen nicht wegen der Mengenausweitung durch die Ärzte in die Höhe, sondern wegen der im Durchschnitt immer weniger gesunden Population. Hier kommen gewichtige weitere Faktoren dazu. Auch jüngere Personen werden durchschnittlich früher krank, einerseits als Folge des Lebensstils mit epidemischer Ausbreitung von Diabetes, Adipositas, Rauchen- und Alkohol- und anderen drogenassoziierten Erkrankungen sowie von Abnützungskrankheiten durch bestimmte Arten der Freizeitbetätigung, insbesondere Sportausübung. Die moderne Ge- 
sellschaft zeichnet sich trotz oder wegen des materiellen Wohlstands durch eine zunehmende Inanspruchnahme psychiatrischer und psychologischer Hilfsangebote aus, während die Seelsorge in den Hintergrund getreten ist.

\section{Der propagierte Wettbewerb}

Die ursprüngliche Idee war, dass durch Wettbewerb die Preise gesenkt werden. Geschehen ist das nicht. Die Preise, nicht aber die Kosten werden nahezu ausschliesslich durch staatliches Diktat gesenkt. Auf Ebene der Krankenkassen ist der Wettbewerb im wesentlichen ein Umverteilungsprozess, der unter dem Strich Prämiengelder verschlingt. Stichwortartig können hierunter alle Aktionen subsumiert werden, in denen es darum geht, die eigene Versichertenstruktur zu optimieren. So werden Versicherungsmodelle angepriesen, die kostengünstiger als andere sind, Hausarzt- und HMO-Modelle, bei denen sich Versicherte verpflichten, keine Spezialisten direkt zu konsultieren. Dieses Managed-CareModell wurde in den USA am weitesten entwickelt. Die USA sind das Land mit den mit Abstand höchsten Gesundheitskosten pro Kopf der Bevölkerung und etwa 40\% der amerikanischen Bevölkerung finden nicht einmal Zugang zu diesem System, weil sie gar nicht versichert sind.

Bekannt ist, dass Versicherungen versuchen, möglichst viele gesunde junge Menschen zu ihren Mitgliedern zu machen. Das geschieht über Sponsoring, womit man sich ein bestimmtes Image als Versicherung verleiht, über Lockvogelangebote wie Beiträge an Fitnesscenterabonnemente usw. Weil aber letztlich die Gesundheitsausgaben durch all diese wettbewerblichen Massnahmen gar nicht beeinflussbar sind, führen diese zu einer Entsolidarisierung, indem diejenigen um so höhere Prämien zahlen, die in keine prämienvergünstigte Versicherungsform eintreten. Würden alle Versicherten einer Versicherung beispielsweise in ein Hausarztmodell gehen, müssten die hier gewährten Rabatte umgehend rückgängig gemacht werden, weil niemand die entstehenden Mindereinnahmen kompensiert. Zudem geht das Hausarztmodell der Krankenkassen von einem schwerwiegenden Irrtum aus. Die Annahme besteht nämlich darin, dass die grundversorgenden Allgemeinmediziner kostengünstiger arbeiten als die Spezialisten. Hierzu gibt es keinerlei stützende Daten. Ob dies tatsächlich so ist, wird davon abhängen, ob die Grundversorger die Weichen richtig stellen. Werden Krankheitszustände richtig eingeschätzt, überhaupt erkannt und vor allem wird erkannt, wann die eigene
Kompetenz ausgereizt ist? Mit anderen Worten: Es kann durchaus günstiger sein, wenn eine Abklärung primär durch einen Spezialisten erfolgt, weil dieser unter Umständen zielgerichteter vorgeht. Ich möchte das an einem Beispiel verdeutlichen: Ein Patient sucht den Hausarzt auf wegen Atemnot. Die physikalische Lungenuntersuchung ergibt keinen bronchospastischen Auskultationsbefund. Dennoch ist der Patient hochgradig obstruktiv («silent chest»). Der Zustand wird verkannt, falsch eingeschätzt und die Weiche falsch gestellt. Hier entstehen unweigerlich Mehrkosten, ganz abgesehen von den gesundheitlichen Folgen für den betroffenen Patienten.

Die Leistungserbringer können Wettbewerb sowieso nicht spielen lassen, denn dieser Part wird durch den Gesetzgeber und die tariflichen Bestimmungen vollständig reguliert. Die Regulierung hat nun auch den Pharmamarkt erfasst.

Gewährte man früher als Wirtschaftspolitiker seinen Partnern in der Wirtschaft allenfalls zuviel Spielraum, so schlägt das nun ins Gegenteil um. So ist der Bundesrat in die «Generikafalle» geraten. Um den Preis von geschätzten 60 Millionen Franken, die durch Umstellung von Originalpräparaten auf Nachahmer einzusparen sind, werden neue indirekte Kosten generiert, die den Spareffekt ins Gegenteil verkehren werden. Diese Kosten werden im wesentlichen als Umstellungskosten zu Buche schlagen, während der sich abzeichnende volkswirtschaftliche Schaden noch gar nicht abschätzbar ist, ist doch die Pharmaindustrie in der Schweiz ein sehr wichtiger Arbeitgeber und Steuerzahler. Flurbereinigungen werden in dem Masse stattfinden, wie die forschende Pharmaindustrie einen verlässlichen staatlichen Partner zu verlieren glaubt. Man muss nur den Blick in unser nördliches Nachbarland wenden, um unschwer zu erkennen, wohin diese staatlichen Eingriffe in den Markt führen. Das Vorgehen ist um so bedenklicher, als es ja der Staat in Form des BSV selber ist, der die Medikamentenpreise dereinst akzeptiert hat. Es ist klar, dass Innovationen von biederen Nachahmern nicht zu erwarten sind und die Schweiz ihre privilegierte Stellung als Testmarkt im positiven Sinn zu verlieren droht.

Nur weil der Wettbewerb im freien Markt funktioniert, heisst das noch lange nicht, dass er in einem derart komplex verschachtelten und regulierten Gesundheitswesen irgendetwas verloren hat. Aber wenn schon der Staat regulierend eingreift, so sollte er allen gleich lange Spiesse gewähren. Genau dies geschieht nicht. 


\section{Wie könnte wirklich gespart werden?}

Es gibt Sparpotential. Richtig eingesetzt wird es die Kostensteigerungen nicht verhindern, jedoch verlangsamen. Würde die Spezialitätenliste der kassenpflichtigen Medikamente nach Kriterien der evidenzbasierten Medizin durchforstet und würde man z.B. verlangen, dass nur noch Mittel mit Wirksamkeitsnachweis der Evidenzklassen I und II (Abb. 1) kassenpflichtig zugelassen bleiben, so bliebe nur noch ein schlankes Büchlein übrig, das in der Kitteltasche Platz findet. Gleiches könnte man tun für andere Leistungen, die im medizinischen Praxisalltag erbracht werden. Insgesamt ist das geschätzte Sparvolumen in dreistelliger Millionenhöhe. Dann müsste darüber nachgedacht werden, welche Leistungen allenfalls unter bestimmten Bedingungen nicht mehr medizinisch sinnvoll sind. Hier müsste ein Konsens gefunden werden, möglichst breit abgestützt. Schliesslich drängen sich Überlegungen auf, wie die zunehmenden Pflegekosten zu finanzieren sind. Die Kopfprämien sind sicher eine schlechte Lösung, denn Pflegeleistungen werden in den kommenden Jahren in immer grösserem Umfang nötig werden. Plafoniert werden dürften sie durch fehlende Pflegeinfrastrukturen und fehlendes Pflegepersonal. Die Nachfrage wird einfach das Angebot übersteigen. Es ist einleuchtend, dass wir das Problem nur werden lösen können, wenn

Abbildung 1

Evidenzklassen: allgemeine Definitionen.

\begin{tabular}{|c|c|}
\hline & $\begin{array}{l}\text { All recommendations provided in this } \\
\text { document follow the format of previous } \\
\text { AcC/AHA guidelines: }\end{array}$ \\
\hline Class I & $\begin{array}{l}\text { Conditians for which there is evidence and/or } \\
\text { general agreement thar a given procedure or } \\
\text { treatment is useful and effective. }\end{array}$ \\
\hline \multirow[t]{3}{*}{ Class II } & $\begin{array}{l}\text { Conditioas for which there is conflicting evidence } \\
\text { and/or a divergence of opinion abour the useful- } \\
\text { ness/efficacy of performing the prosedure/thernapy. }\end{array}$ \\
\hline & $\begin{array}{l}\text { Class Ila Weight of evidencelopinion is in favor } \\
\text { of usefulnessiefficacy. }\end{array}$ \\
\hline & $\begin{array}{l}\text { Class Itb Usefulness/efficacy is less wel established } \\
\text { by evidence/opinion. }\end{array}$ \\
\hline Class III & $\begin{array}{l}\text { Conditions for which there is evideace and/or } \\
\text { general agreement that a procedureitherapy is } \\
\text { nos useful/effective and in some cases may be } \\
\text { harmful. }\end{array}$ \\
\hline
\end{tabular}

die Gesellschaft die Ressourcen der pensionierten Bevölkerung nützen kann, was nur geschehen wird, wenn ein Paradigmenwechsel von den ausrangierten, allenfalls noch eine Zeitlang als Konsumenten willkommenen Pensionierten hin zu wertvollen Mitgliedern der Gesellschaft erfolgt.

\section{Einheitskrankenkasse oder Krankenkassenvielfalt}

Überall in der Wirtschaft finden Konzentrationsprozesse statt. So übernehmen stärkere Unternehmen schwächere, was auch bei den Krankenkassen geschieht. Es sind einige Konglomerate entstanden, die gegeneinander um die Gunst der Kunden buhlen. Sinn macht ein solches System dann, wenn gewisse Rahmenbedingungen erfüllt sind. Erstens muss der Abschluss einer Krankenversicherung freiwillig sein, zweitens muss der Anbieter der Versicherung frei sein in der Gestaltung des Umfangs der Versicherung. Beide Voraussetzungen sind nicht gegeben. Der ganze Wettbewerb verkommt dadurch zum Scheinwettbewerb, da billiger nur der sein kann, der die günstigere Versichertenstruktur aufweist, es sei denn, es werden zu teure Verwaltungskosten generiert. Umgehen lässt sich die sinnlose Verschleuderung von Prämiengeldern für Werbung und Krankenkassenwechsel durch die Abschaffung der Krankenkassen und den Übergang $\mathrm{zu}$ einer gesamtschweizerischen Versicherung. Eigentlich handelt es sich ja nicht um eine Versicherung, sondern um eine Krankenvorsorge. Natürlich wird hier sogleich das Gespenst von einem Desaster, wie es die Invalidenversicherung erfahren hat, sein Unwesen treiben. Aber ganz ungeachtet von demagogischen Versuchen, die Krankenkassenvielfalt zu verteidigen, wird sich mit der Zeit die Überzeugung durchsetzen, dass Krankenkassen anachrone Strukturen sind. Zu erwarten ist, dass ein Konzentrationsprozess wie bei den Banken stattfinden wird. Es ist absehbar, dass dadurch die Macht von Krankenkassen ungesunde Ausmasse annehmen wird.

\section{Ausblick}

Wie kann die skizzierte ungünstige Entwicklung aufgehalten werden? Hier drängt sich als dringlichste KVG-Revision auf, Freiwilligkeit und Eigenverantwortung einzubauen. In einem System staatlich verordneter Zwangsversicherung mit stetig steigenden Prämien wachsen die Begehrlichkeiten der Prämienzahler ins Unermessliche. Mit den vorhandenen Ressourcen wird sorgfältiger umgegangen, wenn eine Eigenleistung gefordert wird. 